\title{
Clinical Study \\ Outcome of Combined Hepatocellular and Cholangiocarcinoma of the Liver
}

\author{
Jue Wang, ${ }^{1}$ Fenwei Wang, ${ }^{2}$ and Anne Kessinger ${ }^{1}$ \\ ${ }^{1}$ Section of Oncology-Hematology, Department of Internal Medicine, University of Nebraska Medical Center, Omaha, \\ NE 68198-7680, USA \\ ${ }^{2}$ Department of Internal Medicine, Creighton University, Omaha, NE 68131, USA
}

Correspondence should be addressed to Jue Wang, juewang@unmc.edu

Received 27 March 2010; Revised 12 June 2010; Accepted 14 July 2010

Academic Editor: Jörg Kleeff

Copyright (C) 2010 Jue Wang et al. This is an open access article distributed under the Creative Commons Attribution License, which permits unrestricted use, distribution, and reproduction in any medium, provided the original work is properly cited.

\begin{abstract}
Background. The objective of this study was to examine the epidemiology, natural history, and prognostic factors of combined hepatocellular and cholangiocarcinoma (cHCC-CC) using population-based registry. Methods. The Surveillance, Epidemiology, and End Results Program database (1973-2004) was used to identify cases of cHCC-CC. Multivariable logistic regression was used to evaluate factors associated with cancer-directed surgery (CDS). The influence of CDS on cancer specific survival was evaluated using Kaplan-Meier curves and Cox proportional hazards modeling. Results. A total of 380 cases of cHCC-CC were identified, which account for approximately $0.87 \%$ of primary liver tumors. Of all patients, $69.8 \%$ of patients had regional or distant stage; $65.6 \%$ of patients had poorly or undifferentiated histology. Only $44.9 \%$ of patients with localized disease, received CDS. By logistic regression analysis, being widowed, advanced stage, and earlier diagnosis year were associated with lower rate of utilization of CDS. In multivariate analysis, tumor stage, receipt of CDS, and recent year of diagnosis were found to be significant predictors for cancer-specific survival. Conclusions. Patients with localized cHCC-CC who are selected for CDS were strongly associated with improved survival. However, many patients with localized tumors did not receive potentially curative cancer-directed surgery. Further study is warranted to address the barriers to the delivery of appropriate care to these patients.
\end{abstract}

\section{Introduction}

Combined hepatocellular and cholangiocarcinoma (cHCC$\mathrm{CC})$ is an uncommon subtype of primary liver cancer [1, 2]. The disease was first described in 1949 by Allen and Lisa and has been defined as the intimate intermingling of both a HCC component and CC component (2). Two histopathological classification schemes have been proposed $(1,2)$. Allen and Lisa [1] described three groups, type A with HCC and CC present at different sites within the same liver, and type B with HCC and CC present at adjacent sites and mingle with continued growth, and type $\mathrm{C}$ with HCC and CC are combined in the same tumor. Goodman et al. [2] categorized cHCC-CC into three types: (i) collision type, (ii) transitional type, and (iii) fibrolamellar type. Kim et al. [3] and Zhang et al. [4] proposed that cHCC-CC is a distinct type of primary liver carcinoma, which is morphologically and phenotypically intermediate between HCC and CC and may be derived from hepatic progenitor cells with the bipotential to differentiate into both hepatocytic and cholangiocytic lineages.

Because of the rarity of cHCC-CC, previously published literature has been based on case series and anecdotal experiences [3-14], with largest case series include thirty cases. The majority case series so far were reported from Asia. Most of these reports were histopathological studies, the demographic features and clinical behavior of this disease remain ill-defined, and the outcomes of these patients are varied in a number of studies. For example, the reported results of surgical resection from single institution have been inconsistent [10, 15-19]. In this study, we take advantage of the vast amount of data collected by the SEER Program to examine the largest series of CHCC-CC reported to date. We examine the incidence, natural history, utilization of cancerdirected surgery (CDS) as well as prognostic factors that might affect the survival for cHCC-CC. 


\section{Methods}

2.1. Data Source. In 1973, the SEER registry program was established to identify all new cancer cases diagnosed within 7 geographic areas [20]. Since 1973, the SEER Program has expanded several times to improve representative sampling of minority groups as well as to increase the total sampling of cases to allow for greater precision. The original SEER 9 registries included Atlanta, Connecticut, Detroit, Hawaii, Iowa, New Mexico, San Francisco-Oakland, Seattle-Puget Sound, and Utah. By 1975, SEER included 9 geographic regions, 5 states (Connecticut, Hawaii, Iowa, New Mexico, and Utah) and 4 metropolitan areas (San Francisco-Oakland, SeattlePuget Sound, Detroit, and Atlanta). In 1992, four additional registries were added to form the SEER 13 registries, which included the SEER 9 registries, plus Los Angeles, San JoseMonterey, rural Georgia, and the Alaska Native Tumor Registry. More recently, in 2000, data from greater California, Kentucky, Louisiana, and New Jersey were added to the SEER 13 Program to form the SEER 17 registries. SEER 9, 13, and 17 registries cover approximately $9.5 \%, 13.8 \%$, and $26.2 \%$ of the total U.S. population, respectively [20]. Data for this study were obtained from SEER* Stat public-use data files available from the National Cancer Institute. For incidence analyses, registry data were linked to total U.S. population data from 1969 to 2003.

2.2. Study Population. The cases of cHCC-CC were extracted from the SEER on the basis of anatomic site (ICD-O-2 codes C22.0-22.1) and histological type (ICD-O code 8180) for those patients first diagnosed and/or treated between January 1973 and December 2004. A total of 42,654 patients with hepatobilliary neoplasms were identified in the SEER 17 registries, among them, 380 patients were found with cHCC-CC. Cases identified at the time of autopsy or by death certificate only ( 24 cases) were excluded from survival analyses.

2.3. Variables. Patients' social demographic characteristics (i.e., age, race/ethnicity, and marital status) and tumor grade and stage at the time of diagnosis, were determined from the SEER database.

Because there is no AJCC (The American Joint Committee on Cancer) staging system for cHCC-CC, general summary stage was used [21]. This system classifies patients as having local, regional (extension into adjacent tissues or nodal involvement), or distant disease. The World Health Organization's standard grading system was used with four separate categories (well, moderately well, poorly differentiated, and undifferentiated). For analysis purpose, they were grouped into low grade (well, moderately well differentiated) and high grade (poorly differentiated, and undifferentiated).

In SEER database, any treatment that is given to modify, control, remove or destroy primary or metastatic cancer tissue is considered to be cancer-directed surgery (CDS). CDS was defined in this study as surgical resection (hepatectomy), transplantation, local regional therapy (such as radiation frequency ablation, chemoembolazation, and embolization), and unknown surgery, based on values for
TABle 1: Age-adjusted incidence rate of combined hepatocellular and cholangiocarcinoma of the liver (cHCC-CC) per 100,000 populations.

\begin{tabular}{lc}
\hline \multicolumn{3}{c}{ Age-adjusted incidence rate per 100,000 (95\% CI $)$} \\
\hline Overall & $0.03(0.02-0.04)$ \\
Men & $0.05(0.03-0.07)$ \\
Women & $0.02(0.01-0.03)$ \\
White & $0.03(0.02-0.04)$ \\
Black & $0.02(0.02-0.04)$ \\
Other & $0.07(0.03-0.12)$ \\
\hline & SEER 13(1992-1999) \\
\hline Overall & $0.03(0.02-0.03)$ \\
Men & $0.04(0.01-0.03)$ \\
Women & $0.02(0.01-0.02)$ \\
White & $0.03(0.02-0.03)$ \\
Black & $0.03(0.00-0.03)$ \\
American Indian & $0.00(0.00-0.03)$ \\
Asian & $0.03(0.00-0.03)$ \\
\hline & \\
\hline Overall & $0.03(0.02-0.03)$ \\
Men & $0.04(0.01-0.03)$ \\
Women & $0.02(0.01-0.02)$ \\
White & $0.03(0.02-0.03)$ \\
Black & $0.03(0.01-0.07)$ \\
American Indian & $0.00(0.00-0.15)$ \\
Asian & $0.03(0.00-0.06)$ \\
\hline
\end{tabular}

SEER: surveillance, epidemiology, and end results.

CI: confidence interval.

site-specific surgery and surgery of the primary site codes within the database [21]. Since only few patients received local regional therapy, we grouped these patients together with patients underwent unknown surgery together as "other surgery".

2.4. Statistical Analysis. By using linked population files, ageadjusted incidence rates and their 95\% confidence intervals (CIs) were calculated for cHCC-CC for all patients, for men and women separately, and for each of the 3 broad categories of race (whites, blacks, and other) and in three time periods.

Discrete data are reported as frequencies and compared by chi-square tests. Continuous data are reported as mean \pm SD and compared by Student's $t$-test. Multivariate logistic regression analyses were used to determine the factors associated with receipt of cancer-directed surgery. Survival duration was measured by the Kaplan-Meier method and compared by the log rank test. Multivariable Cox proportional hazards model was used to identify independent predictors of long-term cancer specific death.

SEER*Stat 6.2.4 (Surveillance Research Program, National Cancer Institute) was used for incidence analyses [20]. All other statistical calculations were performed by SPSS 12.0 (Apache Software Foundation 2000). Survival 
TABLE 2: Characteristics of 380 patients with combined hepatocellular and cholangiocarcinoma of the liver (cHCC-CC) diagnosed between January 1973 and December 2004.

\begin{tabular}{|c|c|c|c|c|}
\hline Characteristics & $N(\%)$ & CDS & No. CDS & $P$ value \\
\hline \multicolumn{5}{|l|}{ Gender } \\
\hline Male & $245(64.5 \%)$ & 49 & 196 & .61 \\
\hline Female & $135(35.5 \%)$ & 30 & 105 & \\
\hline \multicolumn{5}{|l|}{ Race } \\
\hline Black & $43(11.3 \%)$ & 5 & 38 & .09 \\
\hline White & $291(76.6 \%)$ & 60 & 231 & \\
\hline Others & $46(12.1 \%)$ & 14 & 32 & \\
\hline \multicolumn{5}{|l|}{ Civil Status } \\
\hline Married & $222(58.4 \%)$ & 59 & 163 & .01 \\
\hline Divorced/Separated & $37(9.7 \%)$ & 7 & 30 & \\
\hline Single & $52(13.7 \%)$ & 6 & 45 & \\
\hline Widowed & $57(15 \%)$ & 7 & 51 & \\
\hline Unknown & $12(3.2 \%)$ & 0 & 12 & \\
\hline \multicolumn{5}{|l|}{ Grade } \\
\hline Well differentiated & $9(2.4 \%)$ & 1 & 8 & $<.00001$ \\
\hline Moderately differentiated & $34(8.9 \%)$ & 17 & 17 & \\
\hline Poorly differentiated & $70(18.4 \%)$ & 21 & 49 & \\
\hline Undifferentiated & $12(3.2 \%)$ & 2 & 10 & \\
\hline Unknown & $255(67.1 \%)$ & 38 & 217 & \\
\hline \multicolumn{5}{|l|}{ SEER Stage } \\
\hline Localized & $98(25.8 \%)$ & 44 & 54 & $<.0001$ \\
\hline Regional & $97(25.5 \%)$ & 22 & 75 & \\
\hline Distant & $130(34.2 \%)$ & 11 & 119 & \\
\hline Unknown & $55(14.5 \%)$ & 2 & 53 & \\
\hline \multicolumn{5}{|l|}{ Year of diagnosis } \\
\hline 1973-1988 & $154(40.5 \%)$ & 14 & 140 & $<.0001$ \\
\hline 1989-2004 & $226(59.5 \%)$ & 65 & 161 & \\
\hline \multicolumn{5}{|l|}{ Cancer Directed Surgery } \\
\hline Yes & $79(20.8 \%)$ & 79 & 0 & $<.0001$ \\
\hline No & $301(79.2 \%)$ & 0 & 301 & \\
\hline \multicolumn{5}{|l|}{ Radiation Therapy } \\
\hline Yes & $22(5.8 \%)$ & 4 & 18 & .55 \\
\hline No & $354(93.2 \%)$ & 75 & 279 & \\
\hline Unknown & $4(1.0 \%)$ & 0 & 4 & \\
\hline
\end{tabular}

SEER: surveillance, epidemiology, and end results.

durations calculated by SPSS. Comparative differences were considered statistically significant when the $P$ value was $<.05$.

\section{Results}

3.1. Frequency and Incidence. Between 1973 and 2004, a total of 380 patients with cHCC-CC were identified from 42,654 patients with hepatobilliary neoplasms, which consisted of $0.87 \%$ of all patients with hepatobillary cancers. A constant age-adjusted incidence of 0.03 per 100,000 was observed in these three time periods. Detailed incidence data by time period, gender, and race are included in Table 1.
3.2. Patient and Tumor Characteristics. Table 2 includes the patient and tumor characteristics of study cohort. Of the 380 patients with cHCC-CC identified in the SEER database, there was male predominance with a male-to-female ratio of $1.8: 1$. The mean age of patients with cHCC-CC was 64 \pm 12 years (median age 65 years, with a range of 19 to 98 years). The majority of patients (76.6\%) were white. African American accounted for 43 cases (11.03\%). Other ethnicities accounted for 46 cases (12.11\%).

Staging information were not available for 55 (14.5\%) patients. Of the remaining 325 patients, 98 (25.8\%) were classified as localized stage; $97(25.5 \%)$ were classified as regional stage; and $132(34.2 \%)$ were classified as distant stage. 82 of $125(65.6 \%)$ patients whose histology information available had poorly or undifferentiated histology.

3.3. Treatment. Cancer-directed surgery was performed for 79 (20\%) patients, among them, 20 patients underwent liver transplantation, 40 patients underwent partial hepatectomy, 5 patients underwent local surgery (4 patients received radiofrequency ablation (RFA) and 1 patient received percutaneous ethanol injection (PEI)), and the rest of 14 patients underwent unknown surgery. A total of 301 (79.1\%) patients were treated nonsurgically. Radiation therapy was performed in a total $22(5.8 \%)$ of patients, in $4(1.1 \%)$ of 22 patients, radiation was used as an adjuvant to surgery (Table 2).

In a logistic regression analysis, marital status, tumor stage, and year of diagnosis were identified as independent predictors of receiving CDS. The patients who were widowed, patients with advanced stage and those who were diagnosed before 1989, were less likely receiving CDS (Table 3 ). In a separate analysis restricted to patients with local and regional disease, the above factors remained independent predictors of CDS.

3.4. Survival Analysis. The mean followup duration of the entire cohort was 8.4 months. A total of 341 of 380 (89.7\%) patients died during the followup period.

For survival analysis, we excluded the cases that were identified at autopsy or on the basis of death certificates only. A total of 356 patients were included in cancer-specific survival analysis. The median overall survival for all cases was 4 months (95\% CI 3-5).

Figure 1 presents the cancer specific survival rates according to patient and tumor characteristics. Cancerspecific survival rates for entire cohort at 1-, 3-, and 5-year were $26.5 \%, 12.4 \%$, and 9.2\%, respectively, (Figure $1(\mathrm{a})$ ). The 1-, 3-, and 5-year cancer-specific survival rates for patients with local and regional stage tumor were $56.3 \%$, $29.0 \%, 22.1 \%, 25.3 \%$; $9.6 \%$, and $4.8 \%$, respectively, and for patients with distant stage tumor were $6.1 \%, 1.5 \%$, and $0 \%$, respectively, (Figure 1(b)). There was a significant difference in survival between patients who underwent CDS (transplantation, resection or other surgery) versus those did not $(P<.0001)$ (Figure $1(\mathrm{c}))$. The outcomes of patients with cHCC-CC were significantly improved for patients who were diagnosed in later years (1989-2004) compared to those in earlier years (1973-1988) (Figure 1(d)). 


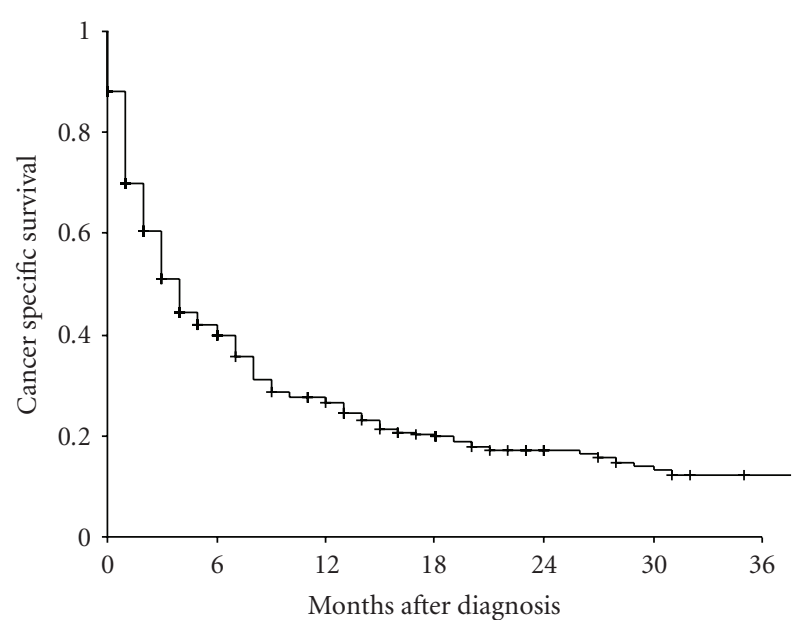

(a)

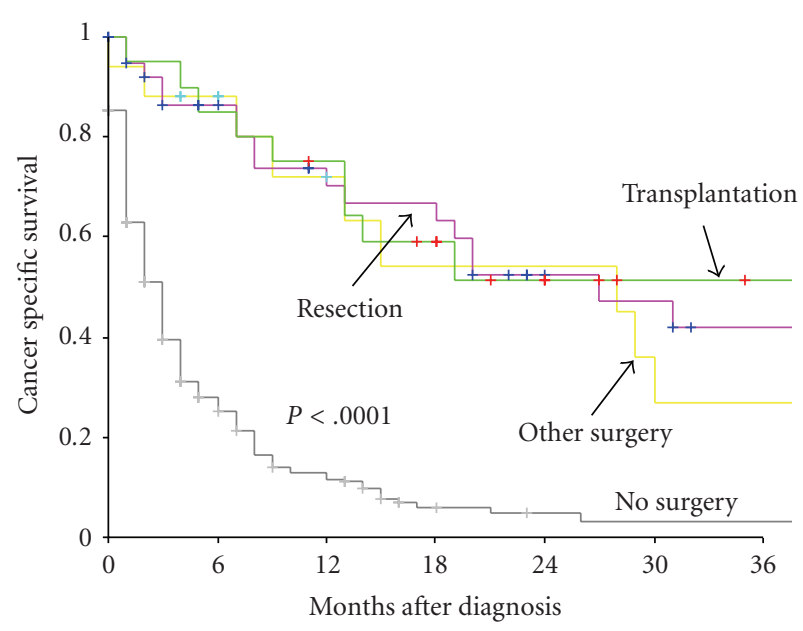

(c)

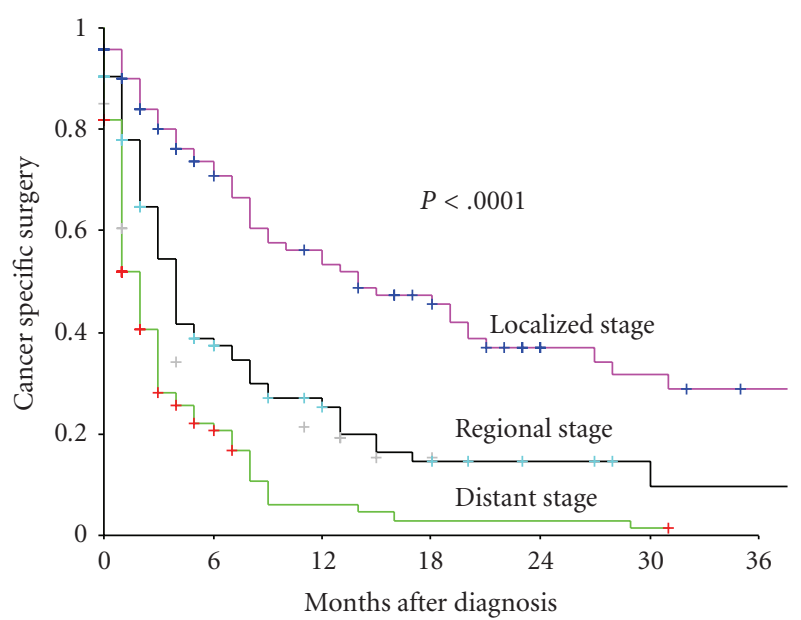

(b)

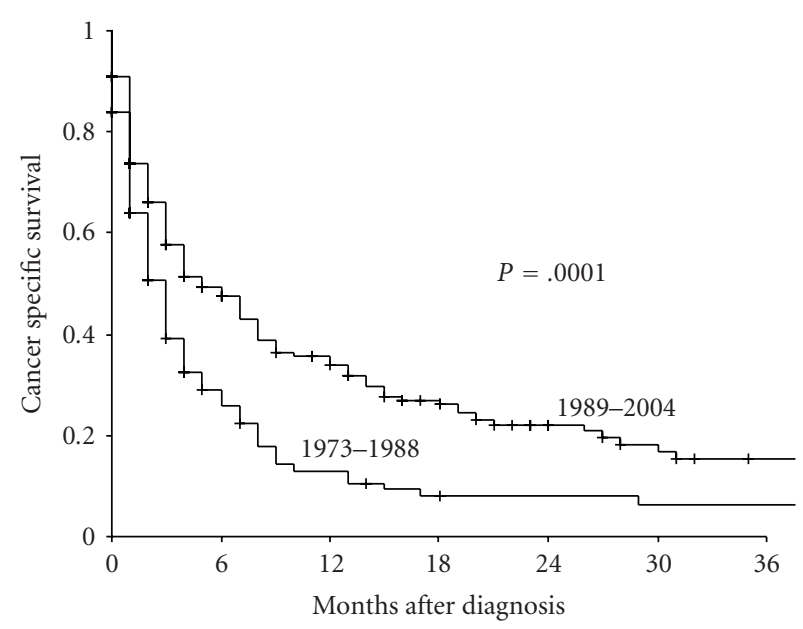

(d)

FIGURE 1: (a) Cancer-specific survival for overall patients with combined hepatocellular and cholangiocarcinoma of the liver (cHCC-CC). (b) Cancer-specific survival rate of patients according to SEER stage. $P<.0001$ for Localized versus Regional versus Distant stage. (c) Cancerspecific survival rate of patients with localized disease according to treatment. $P<.0001$ for transplantation versus resection versus other surgery* versus no surgery. (d) Cancer-specific survival rate of patients according to year of diagnosis. ${ }^{*}$ Other surgery: local surgery plus unknown surgery.

Table 4 presents the result of multivariate survival analyses using Cox proportional hazard model. After adjusting for the demographic, clinical, and treatment-related factors, tumor stage, receiving CDS, and year of diagnosis were identified as independent predictors of cancer-specific survival. Compared to patients with localized disease, patients with regional and distant disease had 1.62 and 2.5 fold increased risk of dying, respectively. The most important predictor of outcome was CDS. Patients who receive cancer directed surgery had significant decrease in the risk of dying than those patients who did not. (Transplantation versus no CDS, HR $=0.25$; hepatectomy versus no CDS, HR $=0.26$; other surgery (local treatment and unknown surgery) versus no CDS, HR = $0.28)$.

\section{Discussion}

The reported frequency of combined tumors in series of primary hepatic malignancies varies widely, from $1.0-4.7 \%$ $[1,2,11,15]$. In this study, cHCC-CC accounted for approximately $0.87 \%$ of primary liver tumors during study period, which is lower than what was reported in single institution studies [13]. "Referral bias" may contribute to the observed higher incidence rates in the single institution studies, which usually were from tertiary hospitals and referral centers. Patients with rare histology subtypes are more likely to visit referral centers for second opinion; pathologists in tertiary centers usually have more opportunity seeing these cases and having expertise to identify these cases, compare with community counterparts. Therefore, the incidence rate 
TAble 3: Multivariate Logistic Regression Analysis of Factors Associated with Cancer Directed Surgery.

\begin{tabular}{lcccc}
\hline Characteristics & Group & OR & $(95 \%$ CI $)$ & $P$ value \\
\hline Age & & 1.00 & $(0.97-1.03)$ & .95 \\
\hline \multirow{2}{*}{ Gender } & Female & 1.00 & & \\
& Male & 0.57 & $(0.29-1.12)$ & .10 \\
\hline \multirow{4}{*}{ SEER Stage } & White & 1.00 & & \\
& Black & 0.33 & $(0.11-1.02)$ & .053 \\
& Other & 0.99 & $(0.43-2.32)$ & .99 \\
\hline \multirow{4}{*}{ Grade } & Localized & 1.00 & & \\
& Regional & 0.32 & $(0.16-0.64)$ & .001 \\
& Distant & 0.12 & $(0.05-0.27)$ & $<.0001$ \\
Civil Status & Unstaged & 0.05 & $(0.01-0.21)$ & $<.0001$ \\
\hline \multirow{2}{*}{ Diagnosis Year } & Low grade & 1.00 & & \\
& High grade & 0.63 & $(0.24-1.63)$ & .34 \\
\hline \multirow{2}{*}{ Radiation } & Unknown & 0.42 & $(0.18-0.99)$ & .05 \\
\hline & Married & 1.00 & & \\
& Widowed & 0.22 & $(0.07-0.66)$ & .007 \\
& 1973-1988 & 1.00 & & \\
\hline \multirow{2}{*}{ 1989-2004 } & 2.90 & $(1.38-6.12)$ & .005 \\
\hline No & 1.00 & & \\
& Yes & 2.60 & $(0.76-8.92)$ & .13 \\
\hline
\end{tabular}

OR: odds ratio; CI: confidence interval; low grade: well differentiated/moderately differentiated; high grade: poorly differentiated/undifferentiated; SEER: surveillance, epidemiology, and end results.

found in a population-based study, which include wide spectrum of hospitals, are more likely to reflect the true incidence of this rare tumor.

Similar with previous studies, most of patients with cHCC-CC presented with high histological grade tumors and advanced stage disease at the time of presentation (Table 2). Our findings, along with others [22], supports the notion that cHCC-CC represents a distinct, aggressive subtype of liver cancer [4-11]. These findings of subtype-related differences in liver cancer have significant implications: better therapy of liver cancer is more likely to be achieved by investigating each subtype of liver cancer separately rather than grouping them all together. As our understanding of the genetic basis for cancer grows, it is likely that liver cancer will be subdivided into ever finer categories.

Surgery is the only treatment offering the possibility of a cure. The main treatment goal should be complete excision with negative margins and limited impact on liver function. In our cohort, only $20 \%$ of all patients received CDS. This likely reflects their advanced stage on presentation. The majority of patients unfortunately were not candidates for potential curative CDS. In addition less than half of the patients with localized disease underwent CDS (Table 2) raises a concern whether cancer-directed surgery was underused in this population. The findings of disparity of CDS utilization in cHCC-CC are similar with findings in studies in HCC [23, 24], efforts should be made
TABLE 4: Cox proportional multivariate analysis of factors associated with cancer-specific mortality.

\begin{tabular}{|c|c|c|c|c|}
\hline Characteristics & Group & HR & $(95 \% \mathrm{CI})$ & $P$ Value \\
\hline Age & & 1.00 & $(0.99-1.01)$ & .56 \\
\hline \multirow{2}{*}{ Gender } & Male & 1.00 & & \\
\hline & Female & 1.27 & $(0.93-1.71)$ & .14 \\
\hline \multirow{3}{*}{ Race } & White & 1.00 & & \\
\hline & Black & 1.41 & $(0.93-2.13)$ & .10 \\
\hline & Others & 1.36 & $(0.91-2.03)$ & .13 \\
\hline \multirow{3}{*}{ SEER Stage } & Localized & 1.00 & & \\
\hline & Regional & 1.59 & $(1.10-2.33)$ & .018 \\
\hline & Distant & 2.50 & $(1.70-3.61)$ & $<.0001$ \\
\hline \multirow{3}{*}{ Grade } & Low & 1.00 & & \\
\hline & High & 1.45 & $(0.90-2.32)$ & .13 \\
\hline & Unknown & 1.02 & $(0.66-1.57)$ & .94 \\
\hline \multirow{5}{*}{ Civil Status } & Married & 1.00 & & \\
\hline & Widowed & 1.21 & $(0.81-1.83)$ & .36 \\
\hline & Divorced/Separated & 0.96 & $(0.59-1.54)$ & .85 \\
\hline & Single & 1.01 & $(0.68-1.49)$ & .96 \\
\hline & Unknown & 0.61 & $(0.31-1.24)$ & .17 \\
\hline \multirow{2}{*}{ Diagnosis Year } & 1973-1988 & 1.00 & & \\
\hline & 1989-2004 & 0.81 & $(0.60-1.09)$ & .16 \\
\hline \multirow{2}{*}{ Radiation } & No & 1.00 & & \\
\hline & Yes & 0.82 & $(0.50-1.38)$ & .47 \\
\hline \multirow{4}{*}{$\begin{array}{l}\text { Cancer Directed } \\
\text { Surgery }\end{array}$} & No & 1.00 & & \\
\hline & Transplantation & 0.25 & $(0.13-0.50)$ & $<.0001$ \\
\hline & Resection & 0.26 & $(0.15-0.44)$ & $<.0001$ \\
\hline & Other Surgery & 0.28 & $(0.15-0.53)$ & $<.0001$ \\
\hline
\end{tabular}

HR: hazard ratio; CI: confidence interval; low grade: well differentiated/ moderately differentiated; high grade: poorly differentiated/undifferentiated; other surgery: local surgery plus unknown surgery; SEER: surveillance, epidemiology, and end results.

to reduce these disparities. The patients diagnosed between 1989 and 2004 were almost three times more likely to receive CDS, in comparison to those diagnosed between 1973 and 1988. This encouraging trend of increasing utilization CDS is in line with the recent advances in radiologic diagnosis $[25,26]$ and a shift towards an earlier stage at diagnosis $[22,27]$, and advances in liver surgery technique $[14,28,29]$.

Although the role of liver transplantation in the management of HCC or cholangiocarcinoma is well defined $[30,31]$, data about the role of liver transplantation in the management of cHCC-CC are lacking [32]. Our study represented the largest series of surgical management of patients with cHCC-CC. At present, liver transplantation and resection are the only potential curative therapy for cHCCCC, therefore, surgery should be considered in patients when complete resection is possible. Because, liver resection carries considerable operative risk; poor performance status and comorbidities may have precluded some patients from resection. 
Journal of Oncology

Currently, there is no literature available addressing impact local regional treatment options on survival specifically on cHCC-CC. Transarterial chemoembolisation (TACE) and percutaneous treatments such as percutaneous ethanol injection (PEI) and radiofrequency ablation (RFA) are widely used treatments for unresectable HCC and postresection recurrence $[33,34]$. However, many cHCCCCs are less vascular and much more fibrotic than HCC, and thus are less likely to respond to TACE or PEI [35]. Our findings suggest RFA is useful in selected patients; however, the small numbers of the patients underwent this therapy and the short duration of followup, makes any conclusions unreliable, and more research is required to confirm this finding.

Consistent with single institution studies, the prognosis of this disease was poor $[15,17,18]$, the median cancer specific survival for the patients with distant disease was 2 months (range 0-31 months). We did, however, observe a significant improvement in the outcome of patients with cHCC-CC over time (Figure 1(d)). Since there was no effective chemotherapy for liver cancer during the study period [36], the better outcomes likely reflect the advance in surgical techniques, better perioperative management and supportive care $[14,28,29]$.

Our findings should be interpreted within the limitations of the study. Although we adjusted for differences in demographic and tumor factors, residual confounding might still be present. Unlike single-institution studies, the accuracy of staging and pathologic diagnosis within a national registry may vary widely across the institutions. In addition, SEER data did not allow us to examine surgical volume, and patient's comorbidities, all of which may influence survival in cancer patients. However, the use of cancer specific survival rather than overall survival in our study has modified the limitation to some degree.

Strengths of this study include the populations-based design and the large sample size. Having large sample size is of particular importance for analysis of rare tumors such as cHCC-CC, where it is nearly impossible for a single institution to collect enough cases to make meaningful analysis regarding important prognostic factors.

\section{Conclusions}

The findings of this study confirmed that CDS was associated with statistically significant increase in cancer-specific survival. However, fewer than $50 \%$ patients with localized disease received CDS. Further study is warranted to explore and address the potential barriers to the delivery of appropriate care to these patients.

\section{Acknowledgments}

The authors acknowledge the efforts of the Applied Research Branch, Division of Cancer Prevention and Population Science, NCI and the Surveillance, Epidemiology, and End Results (SEER) Program tumor registries. Interpretation and reporting of these data are solely the responsibility of the authors.

\section{References}

[1] R. Allen and J. A. Lisa, "Combined liver cell and bile duct carcinoma," American Journal of Pathology, vol. 25, pp. 647$655,1949$.

[2] Z. D. Goodman, K. G. Ishak, and J. M. Langloss, "Combined hepatocellular cholangiocarcinoma. A histologic and immunohistochemical study," Cancer, vol. 55, no. 1, pp. 124135, 1985.

[3] H. Kim, C. Park, K.-H. Han et al., "Primary liver carcinoma of intermediate (hepatocyte-cholangiocyte) phenotype," Journal of Hepatology, vol. 40, no. 2, pp. 298-304, 2004.

[4] F. Zhang, X.-P. Chen, W. Zhang et al., "Combined hepatocellular cholangiocarcinoma originating from hepatic progenitor cells: immunohistochemical and double-fluorescence immunostaining evidence," Histopathology, vol. 52, no. 2, pp. 224-232, 2008.

[5] J. Taguchi, O. Nakashima, M. Tanaka, T. Hisaka, T. Takazawa, and M. Kojiro, "A Clinicopathological study on combined hepatocellular and cholangiocarcinoma," Journal of Gastroenterology and Hepatology, vol. 11, no. 8, pp. 758-764, 1996.

[6] T. Maeda, E. Adachi, K. Kajiyama, K. Sugimachi, and M. Tsuneyoshi, "Combined hepatocellular and cholangiocarcinoma: proposed criteria according to cytokeratin expression and analysis of clinicopathologic features," Human Pathology, vol. 26, no. 9, pp. 956-964, 1995.

[7] Y. Imai, H. Oda, M. Arai et al., "Mutational analysis of the p53 and K-ras genes and allelotype study of the Rb-1 gene for investigating the pathogenesis of combined hepatocellularcholangiocellular carcinomas," Japanese Journal of Cancer Research, vol. 87, no. 10, pp. 1056-1062, 1996.

[8] T. Wakasa, K. Wakasa, T. Shutou et al., "A histopathological study on combined hepatocellular and cholangiocarcinoma: cholangiocarcinoma component is originated from hepatocellular carcinoma," Hepatogastroenterology, vol. 54, no. 74, pp. 508-513, 2007.

[9] H.-Q. Zuo, L.-N. Yan, Y. Zeng et al., "Clinicopathological characteristics of 15 patients with combined hepatocellular carcinoma and cholangiocarcinoma," Hepatobiliary and Pancreatic Diseases International, vol. 6, no. 2, pp. 161-165, 2007.

[10] S. Chantajitr, C. Wilasrusmee, P. Lertsitichai, and N. Phromsopha, "Combined hepatocellular and cholangiocarcinoma: clinical features and prognostic study in a Thai population," Journal of Hepato-Biliary-Pancreatic Surgery, vol. 13, no. 6, pp. 537-542, 2006.

[11] W.-S. Lee, K.-W. Lee, J.-S. Heo et al., "Comparison of combined hepatocellular and cholangiocarcinoma with hepatocellular carcinoma and intrahepatic cholangiocarcinoma," Surgery Today, vol. 36, no. 10, pp. 892-897, 2006.

[12] J. Taguchi, O. Nakashima, M. Tanaka, T. Hisaka, T. Takazawa, and M. Kojiro, "A Clinicopathological study on combined hepatocellular and cholangiocarcinoma," Journal of Gastroenterology and Hepatology, vol. 11, no. 8, pp. 758-764, 1996.

[13] I. O. L. Ng, T. W. H. Shek, J. Nicholls, and L. T. Ma, "Combined hepatocellular-cholangiocarcinoma: a clinicopathological study," Journal of Gastroenterology and Hepatology, vol. 13, no. 1, pp. 34-40, 1998.

[14] R. T. Poon, S. T. Fan, C. M. Lo et al., "Improving perioperative outcome expands the role of hepatectomy in management of benign and malignant hepatobiliary diseases: analysis of 1222 consecutive patients from a prospective database," Annals of Surgery, vol. 240, no. 4, pp. 698-708, 2004. 
[15] W. R. Jarnagin, S. Weber, S. K. Tickoo et al., "Combined hepatocellular and cholangiocarcinoma: demographic, clinical, and prognostic factors," Cancer, vol. 94, no. 7, pp. 2040 2046, 2002.

[16] K. C. Koh, H. Lee, M. S. Choi et al., "Clinicopathologic features and prognosis of combined hepatocellular cholangiocarcinoma," American Journal of Surgery, vol. 189, no. 1, pp. 120-125, 2005.

[17] T. Uenishi, K. Hirohashi, T. Shuto et al., "Surgically treated cases of mixed hepatocellular carcinoma and cholangiocarcinoma," Hepatogastroenterology, vol. 49, no. 46, pp. 1083-1086, 2002.

[18] C.-L. Liu, S.-T. Fan, C.-M. Lo et al., "Hepatic resection for combined hepatocellular and cholangiocarcinoma," Archives of Surgery, vol. 138, no. 1, pp. 86-90, 2003.

[19] L. McCormack, H. Petrowsky, and P.-A. Clavien, "Surgical therapy of hepatocellular carcinoma," European Journal of Gastroenterology and Hepatology, vol. 17, no. 5, pp. 497-503, 2005.

[20] Surveillance, Epidemiology, and End Results (SEER) Program, Public-Use Data (1973-2004), National Cancer Institute, DCCPS, Surveillance Research Program, Cancer Statistics Branch, April 2002, http://www.seer.cancer.gov/ about/expansion.html.

[21] "SEER Summary Staging Manual," 2000, http://seer.cancer .gov/tools/ssm/.

[22] Y. Shiratori, H. Yoshida, and M. Omata, "Management of hepatocellular carcinoma: advances in diagnosis, treatment and prevention," Expert Review of Anticancer Therapy, vol. 1, no. 2, pp. 277-290, 2001.

[23] C. J. Sonnenday, J. B. Dimick, R. D. Schulick, and M. A. Choti, "Racial and geographic disparities in the utilization of surgical therapy for hepatocellular carcinoma," Journal of Gastrointestinal Surgery, vol. 11, no. 12, pp. 1636-1646, 2007.

[24] D. Sloane, H. Chen, and C. Howell, "Racial disparity in primary hepatocellular carcinoma: tumor stage at presentation, surgical treatment and survival," Journal of the National Medical Association, vol. 98, no. 12, pp. 1934-1939, 2006.

[25] Y. Fukukura, J. Taguchi, O. Nakashima, Y. Wada, and M. Kojiro, "Combined hepatocellular and cholangiocarcinoma: correlation between CT findings and clinicopathological features," Journal of Computer Assisted Tomography, vol. 21, no. 1, pp. 52-58, 1997.

[26] S. Phongkitkarun, T. Srisuwan, P. Sornmayura, and J. Jatchavala, "Combined hepatocellular and cholangiocarcinoma: CT Findings with emphasis on multiphasic helical CT," Journal of the Medical Association of Thailand, vol. 90, no. 1, pp. 113-120, 2007.

[27] J. Bruix, A. J. Hessheimer, A. Forner, L. Boix, R. Vilana, and J. M. Llovet, "New aspects of diagnosis and therapy of hepatocellular carcinoma," Oncogene, vol. 25, no. 27, pp. 3848-3856, 2006.

[28] S.-T. Fan, C.-M. Lo, C.-L. Liu et al., "Hepatectomy for hepatocellular carcinoma: toward zero hospital deaths," Annals of Surgery, vol. 229, no. 3, pp. 322-330, 1999.

[29] S. Virani, J. S. Michaelson, M. M. Hutter et al., "Morbidity and mortality after liver resection: results of the patient safety in surgery study," Journal of the American College of Surgeons, vol. 204, no. 6, pp. 1284-1292, 2007.

[30] V. Mazzaferro, E. Regalia, R. Doci et al., "Liver transplantation for the treatment of small hepatocellular carcinomas in patients with cirrhosis," New England Journal of Medicine, vol. 334, no. 11, pp. 693-699, 1996.
[31] C. G. Meyer, I. Penn, and L. James, "Liver transplantation for cholangiocarcinoma: results in 207 patients," Transplantation, vol. 69 , no. 8, pp. 1633-1637, 2000.

[32] A. C.-Y. Chan, C. M. Lo, I. O.-L. Ng, and S. T. Fan, "Liver transplantation for combined hepatocellular cholangiocarcinoma," Asian Journal of Surgery, vol. 30, no. 2, pp. 143-146, 2007.

[33] J. M. Llovet, M. I. Real, X. Montana et al., "Arterial embolisation or chemoembolisation versus symptomatic treatment in patients with unresectable hepatocellular carcinoma: a randomised controlled trial," The Lancet, vol. 359, no. 9319, pp. 1734-1739, 2002.

[34] S. Okada, "Local ablation therapy for hepatocellular carcinoma," Seminars in Liver Disease, vol. 19, no. 3, pp. 323-328, 1999.

[35] E. A. Dick, S. D. Taylor-Robinson, H. C. Thomas, and W. M. W. Gedroyc, "Ablative therapy for liver tumours," Gut, vol. 50, no. 5, pp. 733-739, 2002.

[36] W. T. Kassahun and J. Hauss, "Management of combined hepatocellular and cholangiocarcinoma," International Journal of Clinical Practice, vol. 62, no. 8, pp. 1271-1278, 2008. 


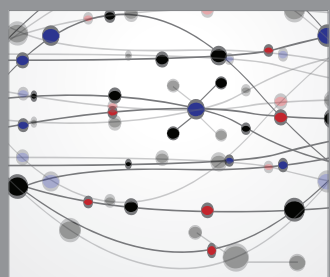

The Scientific World Journal
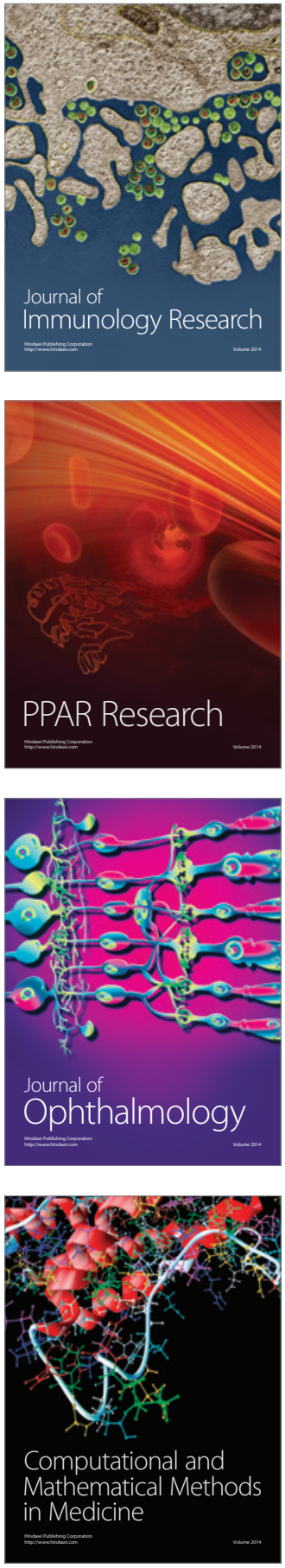

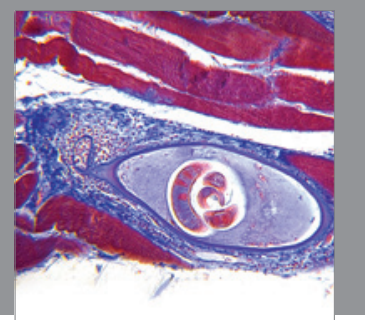

Gastroenterology

Research and Practice
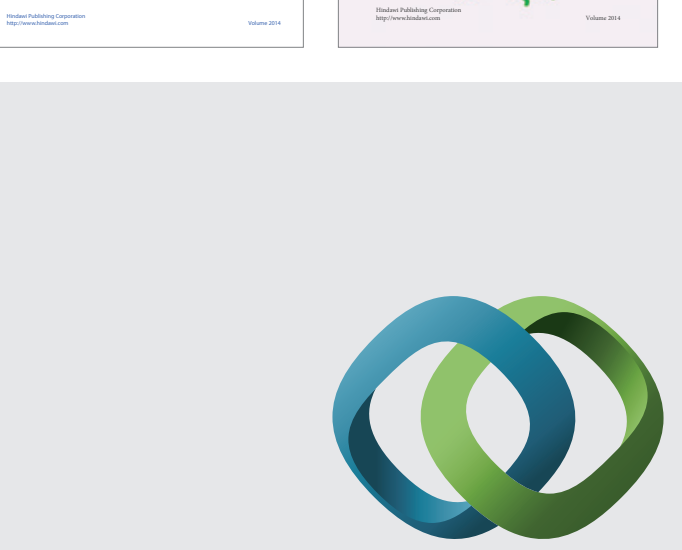

\section{Hindawi}

Submit your manuscripts at

http://www.hindawi.com
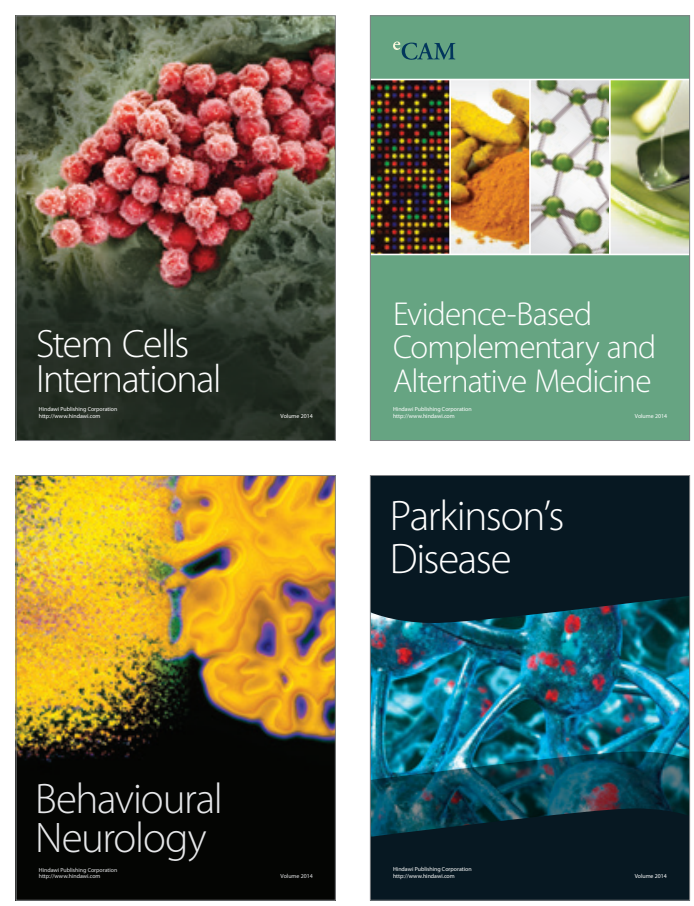

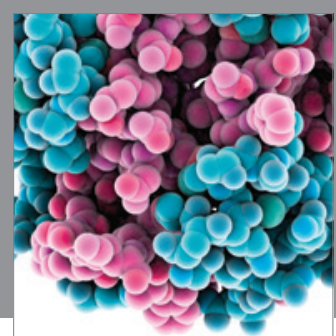

Journal of
Diabetes Research

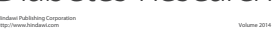

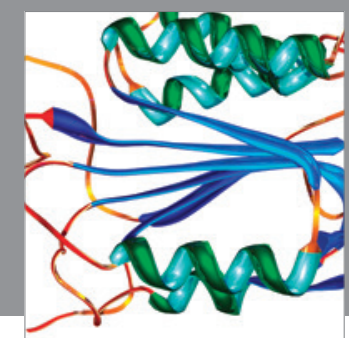

Disease Markers
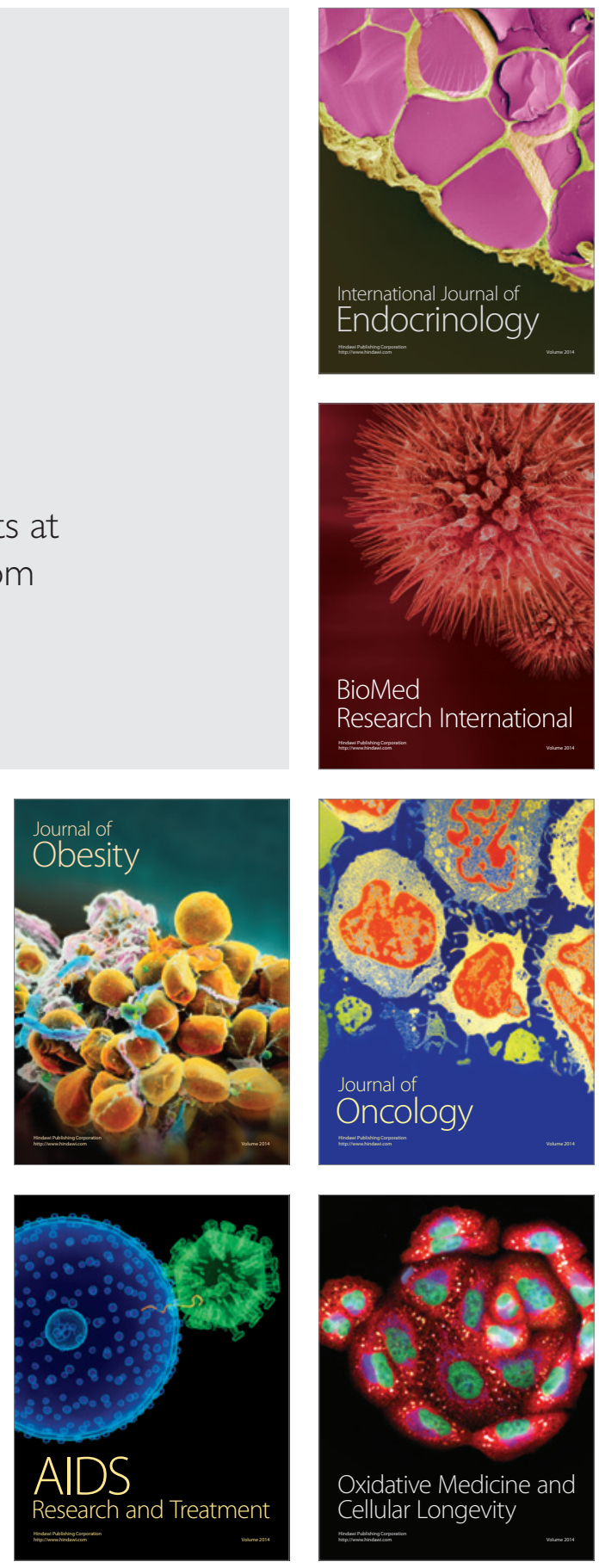